\title{
Design of intelligent and efficient water and electricity cogeneration equipment
}

\author{
Haomin Zhang ${ }^{1 *}, \mathrm{Y}$ uzhe $\mathrm{Wu}^{1}$ \\ ${ }^{1}$ School energy and power engineering, W uhan university of technology, W uhan, Hubei, 430063, China
}

\begin{abstract}
The solar steam generation system studied in this project is mainly composed of a double-layered structure. The upper layer is a nano-fluid material with a light absorber, and the lower layer is an insulating material with two-dimensional or three-dimensional water transport channels. It is hydrophilic to utilize capillary forces and promote Fluid flows to the hot zone, and interconnected pores are used for fluid inflow and outflow to the structure. The device embeds a semiconductor temperature difference power generation sheet in the lower layer, the upper surface is in contact with the photothermal layer, and the lower surface is in contact with the bulk water. The formation of a temperature difference generates an electromotive force, which makes the energy utilization efficiency higher.
\end{abstract}

\section{Introduction}

The global energy crisis and water shortage are one of the most urgent challenges we face. These challenges have prompted scientists to develop various efficient energy conversion and water purification technologies. Among them, solar steam generation and temperature difference power generation are one of the measures to solve the current problems.As irreplaceable freshwater and traditional energy sources such as oil, mines, and natural gas are almost depleted, research and applications on highefficiency solar desalination systems are urgently needed. Solar seawater desalination can directly produce desalinated water through the use of solar energy, and is considered to be one of the irreplaceable technologies to make up for the serious shortage of global water shortages.For the current seawater desalination and temperature difference power generation technologies, the two technologies have common requirements for materials and temperature, so we proposed the idea of organically combining the two. In recent years, various light absorbing materials. ${ }^{[1]}$

In this context, the development of a low-cost, largescale application material that can optimize water evaporation, light absorption performance and reduce heat loss is extremely challenging for efficient and large-scale solar steam generation and water purification Sexual, but also highly desirable.Thermoelectric devices replace traditional heat engines, relying to a large extent on the research and development of thermoelectric materials with higher figure of merit. Low-dimensionalization, gradientization, or element doping can effectively improve the superiority of thermoelectric materials, which is the current research direction of thermoelectric materials. At present, using the phonon scattering mechanism to further reduce the lattice thermal conductivity is an area of research that is of interest. In addition to alloy scattering and grain boundary scattering, phonon scattering mechanisms have recently been proposed, including micro impurity group and nonionized impurity scattering. ${ }^{[2]}$

From the perspective of the market, temperature difference generation technology has the following development advantages:

(1) The energy saving effect is obvious. It is estimated from the utilization of automobile waste heat that the energy consumption of exhaust gas and heat dissipation accounts for about $70 \%$ of the total fuel energy. The conversion efficiency of this material produced by major foreign manufacturers is about $7 \%$, and the energy that can be recovered from it accounts for about the entire fuel. 5\% of the energy, using this part of energy to drive the car can improve the fuel economy of the car by $20 \%$. Nissan company has developed a thermoelectric generator. When the car climbs at a speed of $60 \mathrm{~km} / \mathrm{h}$, the generator can convert $11 \%$ of the heat in the exhaust gas. If the thermoelectric materials can break through in the future, it will be the power supply of future hybrid vehicles The best choice for the system.

(2) Relatively low cost. Since the actual application technology of thermoelectric materials is still in the research stage and the price is relatively high, a single thermoelectric module needs 100 to 250 yuan. If such a thermoelectric generator enters the stage of full industrialization in the future, the cost will be further reduced.

(3) Small size and simple structure. Each piece of thermoelectric module is about $2--4 m$ thick and has an 
area of $16-30 \mathrm{~cm}$. Therefore, the volume occupied by the thermoelectric generator is also limited. The whole device only needs to connect these modules in series and in parallel, and fix them outside the exhaust pipe or the heat collecting plate with a clamping mechanism.

\section{Proposed study plan}

\subsection{Design of double-layer structure of solar steam generation system}

The solar steam generation system studied in this project is mainly composed of a double-layer structure. The design of the double-layer structure is shown in Figure 1. 3 is a hydrophilic heat-absorbing layer that can efficiently perform light-to-heat conversion. It is required to effectively absorb the sun. Light and convert it into heat, which provides the possibility for efficient steam generation; 2 is a hydrophilic insulation layer, as a supporting material, requires low thermal conductivity and good thermal insulation properties to reduce heat loss, you need to have Porosity, there are closed and open pores. Closed pores can help the structure float on the water surface and effectively reduce thermal conductivity, while open pores can provide a channel for water transport, which is conducive to continuous and effective water supply. It has hydrophilicity to use capillary The water is transported to the surface of the absorption layer by force, and at the same time, the weight is required to ensure that the entire structure can float naturally on the water surface; 1 is the water to be purified. The double-layer structure in the device guarantees efficient solar seawater desalination. The temperature difference of the device provides conditions for power generation.

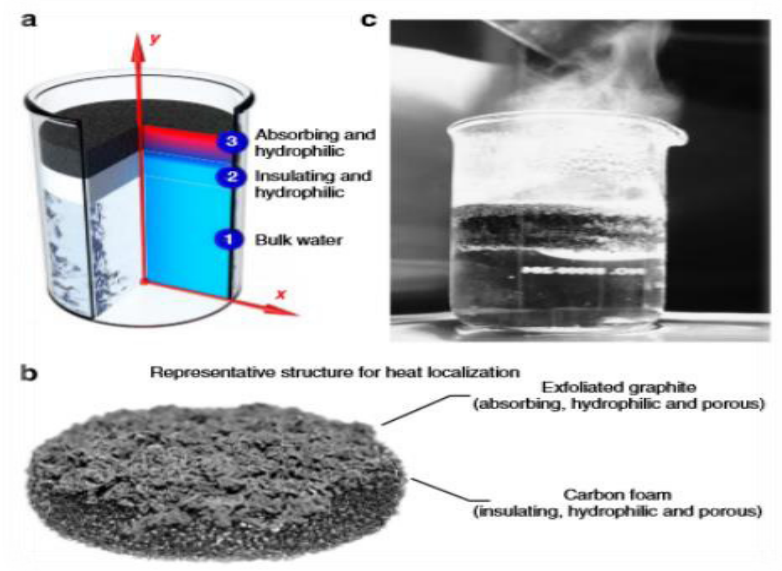

Figure 1.Double-layer structure

\subsection{Semiconductor temperature difference power chip design}

The working principle of a semiconductor temperature difference power chip is to set a temperature difference between two semiconductors with different properties, and a DC voltage is generated across the semiconductors. Temperature difference semiconductor power generation has the characteristics of no noise, long life and stable performance. It can start quickly in the cold environment of minus 40 degrees Celsius, so it is more and more widely used in practice.

The basic principle of thermoelectric power generation is the Seebeck effect: when two metal conductors (or semiconductors) with different free electron densities (or carrier densities) are in contact with each other in a thermoelectric environment, the electrons on the contact surface change from high concentrations to Low concentration diffusion, and the diffusion rate of electrons is directly proportional to the temperature difference in the contact area. Therefore, as long as the temperature difference between the two contacting conductors is maintained, the electrons can continue to diffuse, and a stable voltage is formed between the other two ends of the two conductors. Thermal energy can be directly converted into electrical energy. A semiconductor generator manufactured with a semiconductor temperature difference power generation module can generate electricity as long as there is a temperature difference. No noise, no pollution during work, more than ten years of life, maintenance-free, so it is a widely used portable power supply. Although the current semiconductor thermoelectric power generation is relatively inefficient, with the discovery of various new types of thermoelectric materials with excellent performance and the research on the parameters and structure optimization matching of the thermoelectric generator, the semiconductor thermoelectric power generation technology will be applied in more fields. ${ }^{[3]}$
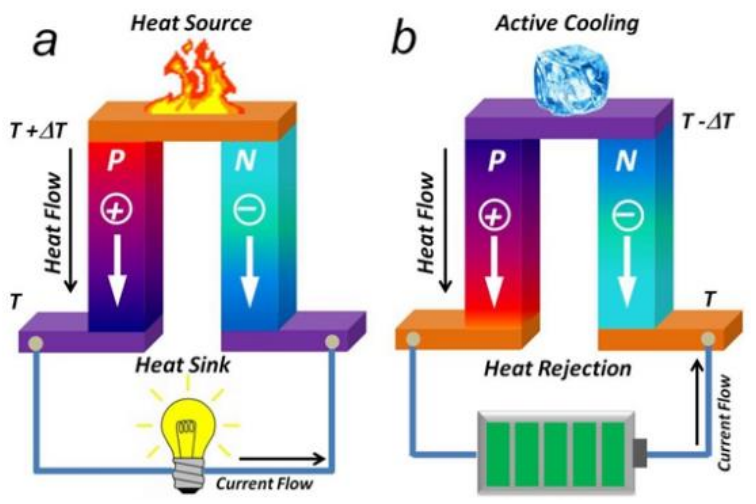

Figure 2. Working principle of semiconductor temperature difference power chip

\section{Project research goals}

The solar steam generation system studied in this project is mainly composed of a double-layered structure. The upper layer is a nano-fluid material with a light absorber, and the lower layer is an insulating material with twodimensional or three-dimensional water transport channels. It is hydrophilic to utilize capillary forces and promote Fluid flows to the hot zone, and interconnected pores are used for fluid inflow and outflow to the structure. The device embeds a semiconductor temperature difference power generation sheet in the lower layer, the upper surface is in contact with the photothermal layer, 
and the lower surface is in contact with the bulk water. The formation of a temperature difference generates an electromotive force, which makes the energy utilization efficiency higher ${ }^{[4]}$.

The foam layer provides buoyancy for the power generation sheet, the water to be purified is transmitted from around the power generation sheet, and the dust-free paper has a water absorption effect. The upper surface of the carbon nanoparticle film attached to the power generating sheet is in contact with the heat absorbing layer, and the lower surface is in direct contact with water, which creates a stable temperature difference with the upper surface. The formation of the temperature difference generates an electromotive force, which makes the energy utilization efficiency higher, and thus better Energy saving.In the context of the global shortage of fresh water, it is very urgent to research an effective solar steam generator. This experimental device is a portable hydropower unit, which is easy to make and convenient to use. Scale promotion. Moreover, the evaporation performance of the device is improved, the heat and mass transfer problems of the microchannel are solved, and the evaporation efficiency of the system is improved.The materials of this experimental device are cheap and easily available.

\section{Innovative and advanced}

The most important innovation and advancement of this project are mainly reflected in:

(1) By studying the micro-nano scale heat and mass transfer problems of the steam generator and the principle of semiconductor temperature difference power generation, the device is optimized for design, using economically available materials, improving the evaporation performance of the entire device, and solving the microchannel Heat and mass transfer issues, improve system evaporation efficiency, and make fuller use of energy.

(2) The use of semiconductor temperature difference power generation has the characteristics of no noise, long life, low input cost, easy preparation and installation, clean and pollution-free, stable performance and so on.

\section{Conclusion}

Based on this project, by studying the micro-nano-scale heat and mass transfer problems of the steam generator and the principle of semiconductor temperature difference power generation, the device is optimized for design, using economically available materials, improving the evaporation performance of the entire device, and solving the problem of microchannels. Heat and mass transfer issues, improve system evaporation efficiency, and make fuller use of energy. Through experimental design, material selection and performance testing were performed on the double-layer structure, and the differences in the comparative materials were determined through characterization. Using comparative experiments as the main line, test the impact of materials on the device. Based on the preliminary completion of the experiments, the software is used to perform simulation analysis of the flow field and thermal field, and calculate and optimize the appropriate parameter ratios, such as porosity, thermal conductivity, and light intensity, to achieve higher evaporation efficiency. Then through experimental verification research, according to the micro-nano scale heat and mass transfer theory, the heat transfer and mass transfer of the entire system is analyzed, calculated and summarized. The optimization of the structure and the improvement of heat and mass transfer performance are completed through experiments.

\section{References}

1. Y u, S.; Zhang, Y .; Duan, H.; Song, C. The impact of surface chemistry on the performance of localized solar-driven evaporation system. Sci. Rep. 2015, 5, 13600.

2. Elimelech, M .; Phillip, W . A . The Future of Seawater Desalination: Energy, Technology, and the Environment. Science 2011, 333, 712-717.

3. Liu Shengguan, Xie Dian, et al. Marine wave, wind and light integrated power ship: China, 201310462964.3[P]. 2013-10-08.

4. Wang, X. Z.; He, Y. R.; Liu, X.; Zhu, J. Q. Enhanced direct steam generation via a bio-inspired solar heating method using carbon nanotube films. Powder Technology. 2017, 321, 276-285.

5. Lin Shigao Liu Xiaolin. The manipulator attitude smoothing algorithm based on the fifth-order polynomial [J]-Manufacturing Automation 2013(21).

6. Ghasemi, H.; Ni, G.; M arconnet, A. M .; Loomis, J .; Yerci, S.; Miljkovic, N.; Chen, G. Solar steam generation by heat localization. Nat. Cummun. 2014, 5,4449 .

7. Li Ru. Discussion on Several Issues of Parallel Operation of Small Diesel Generators. 1999.5 